\title{
Estudo Teórico da Relação Estrutura Atividade Contra o Câncer
}

\author{
Tayane H. Siqueira \& Lilian T. F. M. Camargo
}

As atividades biológicas de 34 derivados análogos da Indolo[2,1b]quinazolina sintetizados foram testadas in vivo e in vitro contra o câncer de pulmão. Com o objetivo de relacionar os parâmetros físico-químicos destes derivados com a atividade anticancerígena, cálculos de mecânica quântica molecular foram realizados usando a teoria do funcional da densidade com o funcional de troca e correlação B3LYP e com o conjunto de base 6-31G* implementados no pacote de programa Gaussian 03. Os descritores obtidos nos cálculos teóricos foram relacionados com a atividade anticancerígena usando métodos da estatística multivariada implementados no programa quimiométrico Einsight. A análise de componentes principais (PCA) mostrou que quatro descritores eletrônicos são suficientes para separar os compostos em duas classes: ativos e inativos. Com base nos descritores selecionados pode se sugerir novos compostos para síntese que sejam mais ativos contra o câncer de pulmão.

\section{Palavras-chave: Palavra chave: Quinazolina, câncer, DFT e PCA}

The biological activities of the Indolo[2,1b]quinalozine and thirty and four analogue compounds were synthesized and tested in vitro against the human lung cancer cells. With the aim to corelate the physical chemical parameters of those compounds with the anticancer activity, quantum molecular calculations were carried out using the Density Functional Theory (DFT) with the exchange correlation functional B3LYP and the basis set $6-31 \mathrm{G}^{*}$ implemented in Gaussian 03 suite of program. The descriptors obtained from the theoretical calculations were related to the anticancer activity using the multivariate statistical methods implemented in the Einsight chemometrics program. The Principal Component Analysis (PCA) showed that four electronic descriptors are able to separate the compounds into two classes: active and inactive. From the selected descriptors it is possible rationally to design new compounds for synthesis that can be more active against lung cancer cells.

Key words: Quinazoline, cancer, DFT e PCA 


\section{Introdução}

O câncer é uma doença que tem assustado a sociedade, pois tem causado muitas mortes em todo o mundo. No Brasil, segundo o INCA estima-se que 466.730 novos casos de câncer surgirão em $2009^{1}$. Esta doença é caracterizada pelo crescimento anormal de células causadas por mudanças na manifestação de características genéticas, levando a um desequilíbrio na proliferação e morte de células, resultando em uma população de células tumorais que podem invadir tecidos e causar metástase a áreas distantes do organismo podendo levar a morte ${ }^{2}$.

As causas do câncer são muito variadas e para vários tipos de câncer elas permanecem desconhecidas, possivelmente estão relacionadas com os fatores externos (o meio ambiente e o estilo de vida) e internos (genéticas) $^{2}$.

O câncer de pulmão é o tipo mais comum de câncer no mundo, neste trabalho estaremos tratando deste tipo de câncer em particular. O padrão da ocorrência desse tipo de neoplasia é determinado por um passado de grande exposição ao tabagismo. Segundo o Instituto Nacional de Câncer (INCA) o câncer de pulmão é uma doença letal. E a taxa de sobrevivência em cinco anos varia de $13 \%$ a $21 \%$ em países desenvolvidos e de $7 \%$ a $10 \%$ em países em desenvolvimento. Verifica-se também que o tabagismo é principal fator de risco para o desenvolvimento dessa neoplasia, em que, os fumantes possuem cerca de 20 a 30 vezes mais riscos de adquirir essa doença do que uma pessoa não fumante ${ }^{1}$.

Atualmente não existe cura para o câncer de pulmão, o tratamento disponível para o seu controle não tem excelente eficácia e, em geral, apresenta efeitos colaterais indesejáveis ${ }^{3}$ por isto cresce o número de pesquisas que visam a formação de novos fármacos ${ }^{4,5,6,7}$ para o combate eficaz desta doença, foi neste esforço que um grupo da Índia sintetizou um grupo de compostos derivados da indolo [2,1B ] quinazolina para ensaios biológicos contra várias linhas de câncer ${ }^{8}$.O presente trabalho pretende investigar usando química quântica computacional e métodos quimiometricos a relação entre a estrutura ${ }^{9} \mathrm{e}$ a atividade anti cancerígena de 34 quinazolinas. Sendo 25 ativas contra o câncer de pulmão e inativas.

\section{Metodologia}

Inicialmente fez-se a análise conformacional dos compostos usando método semi-empírico $\mathrm{PM}^{6}$ implementado no software Hyperchem Release 7.5 ${ }^{10}$ Em seguida as moléculas foram otimizadas usando a Density Functional Theory (DFT) com o funcional de troca e correlação $\mathrm{B} 3 \mathrm{LYP}^{11,12}$ e o conjunto de funções de base $6-31 \mathrm{G}^{*}$ implementados no pacote de programa Gaussian03. ${ }^{13}$ Após a otimização foram calculadas as propriedades moleculares (descritores) para os 34 compostos sob estudo. A Figura 1 mostra a estrutura química da indolo $[2,1 b]$ quinazolina com a respectiva numeração adotada e seus derivados análogos.

Os descritores moleculares calculados neste trabalho com o objetivo de correlacioná-los com a atividade contra o câncer de pulmão foram: $\log \mathrm{P}$; volume $(V)$; polarizabilidade $(\alpha)$; ordens de ligação (Bn-m: ordem de liga) derivadas da análise populacional Natural Bond Order (NBO); ângulos de ligação $\left(\mathrm{A}_{\mathrm{n} \_\mathrm{m} \_\mathrm{p}}\right)$; ângulos

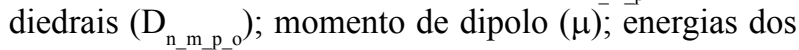
orbitais de fronteira: Highest Occupied Molecular Orbital $\left(\mathrm{E}_{\text {номо }}\right)$ e Lowest Unoccupied Molecular Orbital $\left(\mathrm{E}_{\text {LUMO }}\right)$; dureza $(\eta)$; eletronegatividade de Mulliken $(\chi)$; Gap $\left(\mathrm{E}_{\text {номо }}-\mathrm{E}_{\text {LUмо }}\right)$ e cargas atômicas parciais $\left(c_{n}\right)$ derivada do potencial eletrostático usando o método ChelpG. ${ }^{11}$, Refratibilidae,Energia de Hidratação, e Polarizabilidade.

As estruturas químicas e os valores de $\mathrm{GI}_{50}$ usados neste trabalho foram obtidos de Sharma et al. (2002). ${ }^{6}$ Os compostos podem ser divididos em dois grupos: ativos (1-25), com $\mathrm{GI}_{50}$ até $100 \mu \mathrm{mol} / \mathrm{L}$, e inativos (26-34), com $\mathrm{GI}_{50}$ acima de $100 \mu \mathrm{mol} / \mathrm{L}$.

A correlação entre os descritores calculados e a atividade dos derivados análogos contra o câncer de ovário foi feita usando método da estatística multivariada: Análise de Componentes Principais (PCA) ${ }^{14}$ que do ponto de vista matemático, o PCA é um método que consiste em decompor uma matriz de dados originais $\mathbf{X}$ de posto $r$ em uma soma de $\mathrm{r}$ matrizes de posto 1 , em que o posto é o número de vetores linearmente independentes da matriz X. As matrizes de posto 1 são os produtos dos vetores de scores $\left(S_{h}\right)$ pelos transpostos dos vetores de loadings $\left(l_{h}\right)$, como mostra a Equação 2. ${ }^{15,16}$

$$
\mathbf{X}=\boldsymbol{S}_{1} \cdot \boldsymbol{l}_{1}^{T}+\boldsymbol{S}_{2} \cdot \boldsymbol{l}_{2}^{T}+\cdots+\boldsymbol{S}_{h} \cdot \boldsymbol{l}_{h}^{T}
$$




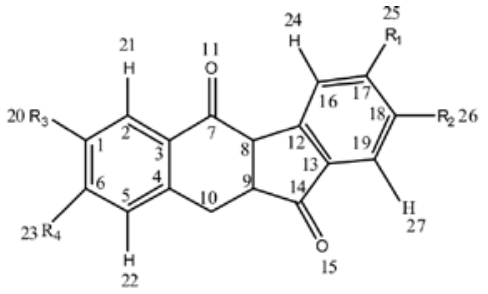

1. $R_{1}=R_{2}=R_{3}=R_{4}=H$

2. $\mathrm{R}_{3}=\mathrm{NH}_{2} ; \mathrm{R}_{1}=\mathrm{R}_{2}=\mathrm{R}_{4}=\mathrm{H}$

3. $\mathrm{R}_{1}=\mathrm{Br} ; \quad \mathrm{R}_{2}=\mathrm{R}_{4}=\mathrm{H}$

4. $\mathrm{R}_{1}=\mathrm{NO}_{2} ; \mathrm{R}_{2}=\mathrm{R}_{3}=\mathrm{R}_{4}=\mathrm{H}$

5. $\mathrm{R}_{1}=\mathrm{NH}_{2} ; \mathrm{R}_{2}=\mathrm{R}_{3}=\mathrm{R}_{4}=\mathrm{H}$

6. $\mathrm{R}_{1}=\mathrm{Me} ; \mathrm{R}_{2}=\mathrm{R}_{3}=\mathrm{R}_{4}=\mathrm{H}$<smiles>[R]Nc1ccc2c(c1)C(=O)c1nc3ccccc3c(=O)n1-2</smiles>

7. $\mathrm{R}=\mathrm{C}(\mathrm{O}) \mathrm{CH}_{3}$

8. $\mathrm{R}=\mathrm{C}(\mathrm{O}) \mathrm{CH}_{2} \mathrm{CH}_{2} \mathrm{CH}_{3}$

9. $\mathrm{R}=\mathrm{C}(\mathrm{O}) \mathrm{CH}_{2} \mathrm{CH}_{3}$

10. $\mathrm{R}=\mathrm{C}(\mathrm{O}) \mathrm{CH}_{2} \mathrm{Cl}$

11. $\mathrm{R}=\mathrm{C}(\mathrm{O}) \mathrm{CH}_{2}$<smiles></smiles>

12. $\mathrm{R}_{1}=\mathrm{R}_{2}=\mathrm{R}_{3}=\mathrm{R}_{4}=\mathrm{R}=\mathrm{H}$

13. $\mathrm{R}_{1}=\mathrm{R}_{2}=\mathrm{R}_{3}=\mathrm{R}_{4}=\mathrm{H} ; \mathrm{R}=-\mathrm{CH}_{2} \mathrm{CH}_{2} \mathrm{OH}$

14. $\mathrm{R}_{1}=\mathrm{R}_{2}=\mathrm{R}_{3}=\mathrm{R}_{4}=\mathrm{H} ; \mathrm{R}=-\mathrm{CH}_{2} \mathrm{COOCH}_{3}$

15. $\mathrm{R}_{1}=\mathrm{R}_{2}=\mathrm{R}_{3}=\mathrm{R}_{4}=\mathrm{H} ; \mathrm{R}=-\mathrm{CH}_{2}$<smiles>CNc1ccccn1</smiles>

16. $\mathrm{R}_{1}=\mathrm{R}_{2}=\mathrm{R}_{3}=\mathrm{R}_{4}=\mathrm{H} ; \mathrm{R}=-\mathrm{C}(\mathrm{O}) \mathrm{CH}=\mathrm{CH}-\mathrm{Ph}$

17. $\mathrm{R}_{1}=\mathrm{R}_{2}=\mathrm{R}_{3}=\mathrm{R}_{4}=\mathrm{H} ; \mathrm{R}=-\mathrm{CH}_{2} \mathrm{CH}_{2} \mathrm{CH}_{2}-\mathrm{NMe}_{2}$

18. $\mathrm{R}_{1}=\mathrm{R}_{2}=\mathrm{R}_{3}=\mathrm{R}_{4}=\mathrm{H} ; \mathrm{R}=-\mathrm{CH}_{2} \mathrm{CH}_{2} \mathrm{CH}_{2}-\mathrm{NMe}_{2}$

$19 . \mathrm{R}_{1}=\mathrm{R}_{2}=\mathrm{R}_{3}=\mathrm{R}_{4}=\mathrm{H} ; \mathrm{R}=-\mathrm{CH}_{2}$

20. $\mathrm{R}_{1}=\mathrm{R}_{2}=\mathrm{R}_{3}=\mathrm{R}_{4}=\mathrm{H} ; \mathrm{R}=-\mathrm{CH}_{2} \backslash$

21. $\mathrm{R}_{1}=\mathrm{NO}_{2} ; \mathrm{R}_{2}=\mathrm{R}_{3}=\mathrm{R}_{4}=\mathrm{H} ; \mathrm{R}=-\mathrm{CH}_{2} \mathrm{CH}_{2}-\mathrm{NMe}_{2}$

22. $\mathrm{R}_{1}=\mathrm{NO}_{2} ; \mathrm{R}_{2}=\mathrm{R}_{3}=\mathrm{R}_{4}=\mathrm{H} ; \mathrm{R}=-\mathrm{CH}_{2} \mathrm{CH}_{2} \mathrm{CH}_{2}-\mathrm{NMe}_{2}$

23. $\mathrm{R}_{1}=\mathrm{NO}_{2} ; \mathrm{R}_{2}=\mathrm{R}_{3}=\mathrm{R}_{4}=\mathrm{H} ; \mathrm{R}=-\mathrm{CH}_{2} \mathrm{CH}_{2} \mathrm{OH}$

24. $\mathrm{R}_{1}=\mathrm{Br} ; \mathrm{R}_{2}=\mathrm{R}_{3}=\mathrm{R}_{4}=\mathrm{H} ; \quad \mathrm{R}=-\mathrm{CH}_{2} \mathrm{CH}_{2}-\mathrm{NMe}_{2}$

25. $\mathrm{R}_{1}=\mathrm{Br} ; \mathrm{R}_{2}=\mathrm{R}_{3}=\mathrm{R}_{4}=\mathrm{H} ; \mathrm{R}=-\mathrm{CH}_{2}$<smiles>[R]c1cc2c(cc1[R])C(=O)n1c(nc3cc([R])c([R])cc3c1=O)C2=O</smiles>

26. $\mathrm{R}_{3}=\mathrm{Br} ; \mathrm{R}_{1}=\mathrm{R}_{2}=\mathrm{R}_{4}=\mathrm{H}$

27. $\mathrm{R}_{3}=\mathrm{NO}_{2} ; \mathrm{R}_{1}=\mathrm{R}_{2}=\mathrm{R}_{3}=\mathrm{R}_{4}=\mathrm{H}$

28. $\mathrm{R}_{3}=\mathrm{R}_{4}=\mathrm{OMe} ; \mathrm{R}_{1}=\mathrm{R}_{2}=\mathrm{H}$

29. $\mathrm{R}_{1}=\mathrm{R}_{2}=-\mathrm{OCH}_{2^{-}} ; \mathrm{R}_{3}=\mathrm{R}_{4}=\mathrm{H}$

$30 . \mathrm{R}_{1}=\mathrm{OMe} ; \mathrm{R}_{2}=\mathrm{R}_{3}=\mathrm{R}_{4}=\mathrm{H}$

31. $\mathrm{R}=-\mathrm{CH}_{3}$

32. $\mathrm{R}=-\mathrm{CH}_{2} \mathrm{CH}_{3}$

33. R $=-\mathrm{CH}_{2} \mathrm{CH}_{2} \mathrm{CH}_{2} \mathrm{CH}_{3}$

$34 . \mathrm{R}=-\mathrm{OCH}_{2} \mathrm{CH}_{2} \mathrm{O}-\mathrm{Ph}$

Figura 1: Estruturas moleculares e numeração atômica adotada nos cálculos da Indolo[2,1B] quinazoline e seus derivados análogos. 
A principal vantagem no uso da análise de componentes principais é a possibilidade de diminuir a dimensão dos dados originais.

\section{Resultados e Discussão}

Foi necessário autoescalar os dados uma vez que as variáveis possuíam diferentes escalas, sendo que no autoescalamento cada variável é escalada para ter média zero e variância unitária. ${ }^{16}$ Após inúmeras combinações de variáveis, a melhor separação foi obtida usando os descritores $\mathrm{B}_{5 \_22}, \mathrm{~B}_{9 \_14}, \mathrm{~B}_{12 \_13}, \mathrm{~B}_{17 \_25}$, (onde $B$ representa ordem de ligação). Os valores destas variáveis são mostrados na Tabela 2 .

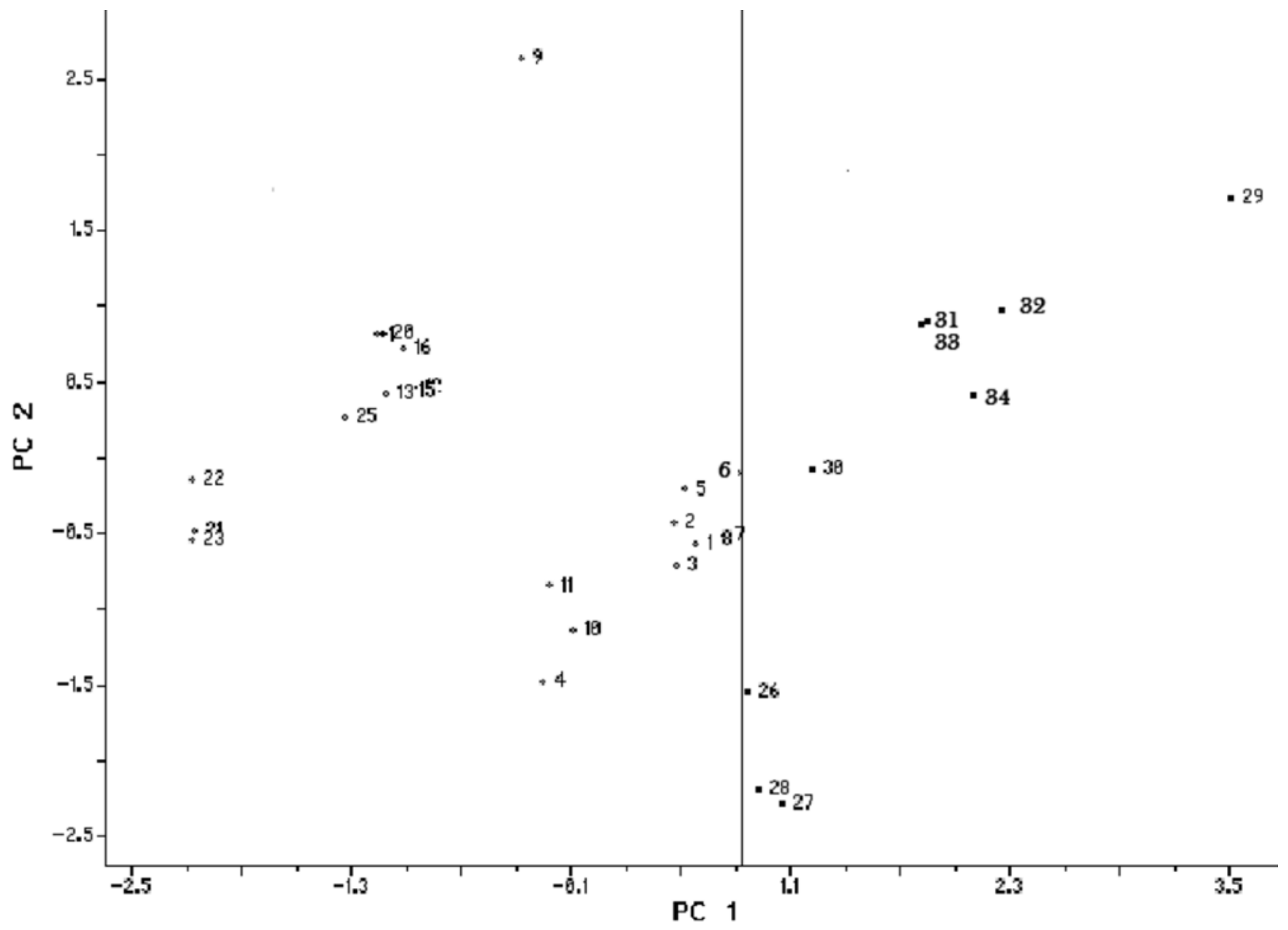

Tabela 1 - Valores das ordens de ligação responsáveis pela discriminação das classes a nível de teoria B3LYP/6-31G*.

\begin{tabular}{ccc}
\hline Componentes & Percentagem Individual & Percentagem cumulada \\
\hline PC_01 & 48,2270 & 48,2270 \\
PC_02 & 26,9171 & 75,1441 \\
PC_03 & 20,2786 & 95,4228 \\
\hline
\end{tabular}

A Figura 2 mostra que a primeira componente (PC1) é responsável pela discriminação entre compostos ativos (1-25) e inativos (26-34). Sozinha, esta componente explica 48,23\% da variância total dos dados. As duas primeiras componentes explicam junta $75,14 \%$. Considerando as três primeiras componentes, 95,42\% da variância dos dados são explicadas. Na Tabela 3 está indicado os pesos das variáveis responsáveis pela separação.

Figura 2: Representação gráfica dos scores obtidos usando o programa Einsight 3.0. 
A Equação 3 mostra a influência dos pesos no cálculo dos scores da PC1, a qual foi responsável pela discriminação das classes em ativa e inativa.

$$
\mathrm{PC} 1=-0,19\left(\mathrm{~B}_{5 \_22}\right)-0,62\left(\mathrm{~B}_{9 \_14}\right)+0,65\left(\mathrm{~B}_{12 \_13}\right)+0,40\left(\mathrm{~B}_{17 \_25}\right)
$$

Com base na Equação 3 para um composto pertencer ao grupo dos compostos ativos deve apresenta altos valores numéricos das variáveis $\left(\mathrm{B}_{91_{14}}\right.$ e $\left.\mathrm{B}_{522}\right)$ e baixos valores numéricos das variáveis $\left(\mathrm{B}_{12 \_13}\right.$ e $\left.\mathrm{B}_{17 \_25}\right)$. Analisando os dados da Tabela 3, observa-se que a ordem de ligação com maior peso na separação das variáveis foi a ordem de ligação entre os átomos 12 e 13 (veja Figura 1) e a variável de menor peso na primeira componente foi a ordem de ligação entre os átomos 5 e 22.

Tabela 2 - Pesos das variáveis na primeira e segunda componente.

\begin{tabular}{rccc}
\hline Variáveis & PC_01 & PC_02 & PC_03 \\
\hline B5.22 & $-0,19$ & 0,85 & 0,41 \\
B9.14 & $-0,62$ & 0,24 & 0,37 \\
B12.13 & 0,65 & 0,23 & 0,25 \\
B17.25 & 0,40 & 0,40 & 0,80 \\
\hline
\end{tabular}

Aplicando a técnica de quimiometria: Análise de Componentes Principais (PCA), foi selecionados os descritores calculados teoricamente que estão correlacionados com a atividade contra o câncer de mama. As variáveis que melhor discriminaram as moléculas ativas e inativas foram as ordens de ligação entre os átomos (Figura 1): 5-22, 9-14, 12-13 e 17-25. Com base nos resultados, é possível mapear indicativos para a síntese novos compostos que sejam ativos contra o câncer de ovário, ou seja, é necessário aumentar o valor numérico das variáveis $\left(\mathrm{B}_{522}\right.$ e $\left.\mathrm{B}_{914}\right)$ e diminuir o valor numérico das variáveis $\left(\mathrm{B}_{12 \_13}\right.$ e $\left.\mathrm{B}_{17 \_25}\right)$ que foram significativas na separação das moléculas quanto à sua atividade.

\section{BIBLIOGRAFIA}

1. Brasil; Estimativa 2008: Incidência de Câncer no Brasil. INCA: Rio de Janeiro, 2007.
2. Ruddon, R. W; Cancer biology, 4th. Oxford University Press: Michigan, 2007.

3. Temmink, O. H.; Prins, H. J, Van Gelderop, E., Peters, G. J.; Brit. J. of canc., 2007, 96, 61.

4. M. V. Ramana Reddy, Muralidhar R. Mallireddigari, Stephen C. Cosenza, Venkat R. Pallela, Nabisa M. Iqbal,Kimberly A. Robell, Anthony D. Kang, And E. Premkumar Reddy. Design; J. Med. Chem., 2008, 51, 86.

5. Kyeong Lee, A Jung S. Ryu,B Yinglan Jin,A Woncheol Kim,A Navneet Kaur,A Sang J. Chung,A Yong-Jin Jeon,Bjoon-Tae Park,B JI S. Bang,B Hong S. LEE,B Tae Y. Kim,B Jung J. Leea AND Young-soo Hong; Organic \& Biomolecular Chemistry. 2008.

6. Vedula M. Sharma,* K. V. AdI Seshu, V. Chandra Sekhar,Sachin Madan,B. VIshnu, P. Aravind Babu,C. Vamsee Krishna, J. Sreenu, V. Ravi Krishna,A. Venkateswarlu, Sriram Rajagopal, R. Ajaykumar and T. Sravan Kumar. Bioorganic \& Medicinal Chemistry Letters, 2004, 14, 67.

7. VedulaM.Sharma, ${ }^{*}$ K.V.adiSeshu,C.VamseeKrishna, P. Prasanna,V. Chandra Sekhar, A. Venkateswarlu, Sriram Rajagopal, R. Ajaykumar,Dhanvanthri S. Deevi, N. V. S. Rao Mamidi and R. Rajagopalan; Bioorganic \& Medicinal Chemistry Letters, 2003, 13 1679.

8. Sharma, V.M.; Prasanna, P.; Seshu,k.V.A.; Renuka,B. Bioorganic \& Medicinal Chemistry. 2002, 12, 2303.

9. Mati, K.; Labonov, V.S. J. Amer. Chem. Soc. 1996, 96, 1027.

10. Hyperchem, Release 7.0, Hypercube Inc. 1115 NW 4th Street, Gainesville, FL 32601, USA.

11. Lee, C.; Yang, W.; Parr, R. G.; Phys. Rev. 1988, B37, 785.

12. Becke, A. D.; Phys. Rev. 1988, A38, 3098.

13. Frisch, M. J.; Trucks, G. W.; Schlegel, H. B.; Scuseria, G. E.; Robb, M. A.; Cheeseman, J. R., J. A. Montgomery, JR.; Vreven T.; Kudin K. N.; Burant, J. C.; Millam, J. M.; Iyengar S. S.; Tomasi J.; Barone, V.; Mennucci, B.; Cossi, M.; Scalmani, G.; Rega, 
N.; Petersson G. A.; Nakatsuji, H.; Hada, M.; Ehara, Toyota, M., K.; Fukuda, R.; Hasegawa, J.; Ishida, M.; Nakajima, T.; Honda, Y.; Kitao, O.; Nakai, H.; Klene, M., Li, X.; Knox, J. E.; Hratchian, H. P.; Cross, J. B.; Adamo, C., Jaramillo, J.; Gomperts R.; Stratmann, R. E.; Yazyev, O.; Austin, A. J.; Cammi, R.; Pomelli, C.; Ochterski, J. W.; Ayala, P. Y.; Morokuma K.; Voth, G. A.; Salvador, P.; Dannenberg, J. J., Zakrzewski, V. G.; Dapprich, S.; Daniels, A. D.; Strai,N M. C.; Farkas, O.; Malick, D. K.; Rabuck, A. D.; Raghavachari, K.; Foresman, J. B.; Ortiz, J. V.; Q. CUI, Baboul, A. G.; Clifford, S.; Cioslowski, J.; Stefanov, B. B.; Liashenko, G. LIU, A.; Piskorz P., Komaromi, I.; Martin R. L.; Fox, D. J.; Keith, Al-laham, T. M. A.; Peng, C. Y.; Nanayakkara, A.; Challacombe, M.; Gill, P. M. W.; Johnson, B.; Chen, Wong, W.; Gonzalez, M. W.; C.; Pople, J. A.; Gaussian 03, Revision B.04, gaussian, inc., Pittsburgh pa, 2003.
14. Press, S.J. Applied multivariate statistics. Holts, Rinehart and Winston: New York, 1972.

15. Massart, D. L.; Chemometrics tutorial. Amsterdam, Elsevier, 1990.

16. Magalhães, D.; Bruns, R. E.; Vasconcellos, P. C. Quim. Nova, 2007, 30, 577.

\section{Tayane H. Siqueira', Lilian T. F. M. Camargo*1}

${ }^{1}$ Faculdade de Tecnologia SENAI Roberto Mange, CP 355, CEP 75113-630, Anápolis, GO, Brasil

*E-mail: lilianthaty@yahoo.com.br 